\title{
Extra dimensions and Higgs pair production at photon colliders
}

\author{
Xiao-Gang $\mathrm{He}^{*}$ \\ Department of Physics, National Taiwan University, Taipei, Taiwan 10617, Republic of China
}

(Received 22 July 1999; published 12 November 1999)

\begin{abstract}
We show that new physics effects due to extra dimensions can dramatically affect Higgs pair production at photon colliders. We find that the cross section due to extra dimensions with the scale $M_{S}$ of new physics around $1.5 \mathrm{TeV}$ can be as large as $0.11 \mathrm{pb}(1.5 \mathrm{pb})$ for monochromatic photon collision, $\gamma \gamma \rightarrow H H$, with the collider energy $\sqrt{s}=0.5(1) \mathrm{TeV}$ for a Higgs boson mass of 100 (350) GeV. The cross section can be $3 \mathrm{fb}(2.7$ $\mathrm{fb}$ ) for the same parameters for collisions using photon beams from electron or positron back scattered by laser. These cross sections are much larger than those predicted in the standard model. Higgs pair production at photon colliders can provide useful tests for new physics due to extra dimensions. [S0556-2821(99)00723-7]

PACS number(s): 12.60.Fr, 14.80.Cp
\end{abstract}

It has recently been proposed that gravitational effects can become large at a scale $M_{S}$ near the weak scale due to effects from extra dimensions $[1,2]$, which is quite different from the traditional thought that gravitational effects only become large at the Planck scale $M_{P l} \sim 10^{19} \mathrm{GeV}$. In this proposal the total space-time is $D=4+n$ dimensions. When the extra dimensions are compactified there are towers of states, the Kaluza-Klein (KK) states, that interact with ordinary matter fields. Although the interaction with the standard model (SM) fields for each of the KK state is small, proportional to the Newton constant $G_{N}$ the effects become much stronger, proportional to $1 / M_{S}^{2}$, when the contributions of all the KK states are summed over.

The relation between the scale $M_{S}$ and the Planck scale $M_{P l}$, assuming all extra dimensions are compactified with the same size $R$, is given by $M_{P l}^{2} \sim R^{n} M_{S}^{2+n}$. With $M_{S}$ near a $\mathrm{TeV}$, for $n=1 R$ would be too large so this theory is ruled out. However, with $n$ larger than or equal to 2 , the theory is not ruled out. In these cases, $R$ can be in the submillimeter region which can be probed by laboratory experiments [3-8]. The lower bound for $M_{S}$ is constrained, typically, to be of order one $\mathrm{TeV}$ from present experimental data [3-8]. Future experiments will provide more stringent constraints. It is important to investigate as many systems as possible to give constraints on the scale $M_{S}$ and to look for deviations from predictions in the SM to isolate effects due to extra dimensions. In this paper we study effects due to extra dimensions on Higgs pair production at photon colliders. We find that the cross section for this process can be dramatically different from SM predictions.

In the minimal standard model there is a neutral Higgs boson $H$ resulting from spontaneous symmetry breaking of $\mathrm{SU}(2)_{L} \times \mathrm{U}(1)_{Y}$ to $\mathrm{U}(1)_{e m}$ due to the Higgs mechanism. The mechanism for spontaneous symmetry breaking is not well understood. There is no experimental evidence favoring any particular mechanism, such as the Higgs mechanism. The discovery of the Higgs boson and understanding of its properties are fundamentally important [9]. The Higgs boson is also the last particle yet to be discovered in the minimal

*Email address: hexg@phys.ntu.edu.tw
SM. The discovery of the Higgs boson is one of the most important goals for future high energy colliders. At present the lower bound on the SM Higgs boson mass $m_{h}$ is set by the CERN $e^{+} e^{-}$collider LEP II to be $95.5 \mathrm{GeV}$ at $95 \%$ C.L. [10]. Many methods have been proposed to produce and to study the properties of Higgs bosons [9]. Higgs pair production at various colliders are some of the interesting ones [1113]. In particular Higgs pair production through $\gamma \gamma \rightarrow H H$ is important for the study of Higgs boson properties. In SM the process $\gamma \gamma \rightarrow H H$ only occurs at loop level with cross section of order $\mathrm{O}(0.5 \mathrm{fb})$ for $\sqrt{s} \sim(500$ to 1000$)$ and $m_{h}$ in the range 100 to $400 \mathrm{GeV}$ [12]. It is in principle reachable [14], but a formidable challenge for accelerator physics. The smallness of cross section predicted in SM makes the process, $\gamma \gamma \rightarrow H H$, sensitive to new physics beyond the minimal SM. New physics beyond SM may dramatically change the situation and increase the cross section to a more practical level for experiments [13]. In the following we show that this is indeed possible with extra dimensions.

After compactifying the extra $n$ dimensions, for a given KK level $\vec{l}$ there is one spin- 2 state, $n-1$ spin- 1 state, and $n(n-1) / 2$ spin-0 states [7]. Assuming that all SM fields are confined to a four dimensional world volume and gravitation is minimally coupled to SM fields, it was found that the spin-1 KK states decouple while the spin-2 and spin-0 KK states couple to all SM fields [7]. Since the KK states interact with particles in the SM, exchanges of KK states can generate new interactions among the SM particles. For $\gamma \gamma \rightarrow H H$ we find that, contrary to the situation in SM, this process can happen at the tree level due to exchange of spin-2 KK states as shown in Fig. 1. Spin-1 and spin-0 KK states do not

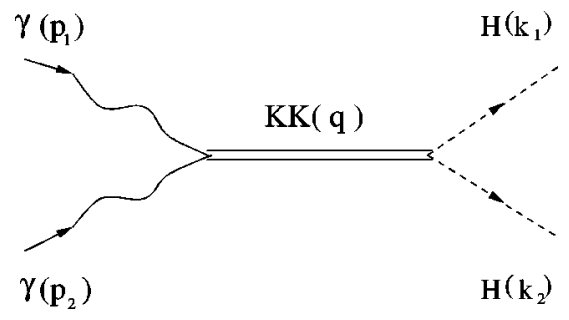

FIG. 1. Tree diagram for $\mathrm{KK}$ states contribution to $\gamma\left(p_{1}\right) \gamma\left(p_{2}\right) \rightarrow H\left(k_{1}\right) H\left(k_{2}\right)$. 
contribute to this process. Using the Feynmann rules given in Ref. [7], we have

$$
\begin{aligned}
\tilde{M}(\gamma \gamma \rightarrow & H H)=\frac{\kappa^{2}}{8}\left(m_{h}^{2} \eta^{\mu \nu}-C^{\mu \nu, \rho \sigma} k_{1 \rho} k_{2 \sigma}\right) \\
& \times \frac{B_{\mu \nu, \alpha \beta}}{q^{2}-m_{l}^{2}}\left(p_{1} \cdot p_{2} C^{\alpha \beta, \delta \gamma}+D^{\alpha \beta, \delta \gamma}\right) \epsilon_{1 \delta}\left(p_{1}\right) \epsilon_{2 \gamma}\left(p_{2}\right), \\
C^{\mu \nu, \rho \sigma=} & \eta^{\mu \rho} \eta^{\nu \sigma}+\eta^{\mu \sigma} \eta^{\nu \rho}-\eta^{\mu \nu} \eta^{\rho \sigma}, \\
D^{\alpha \beta, \delta \gamma=} & \eta^{\alpha \beta} k_{1}^{\delta} k_{2}^{\gamma}-\left[\eta^{\alpha \gamma} k_{1}^{\beta} k_{2}^{\delta}+\eta^{\alpha \delta} k_{1}^{\gamma} k_{2}^{\beta}-\eta^{\delta \gamma} k_{1}^{\alpha} k_{2}^{\beta}\right. \\
& +(\alpha \rightarrow \beta, \beta \rightarrow \alpha)], \\
B_{\mu \nu, \alpha \beta}= & \left(\eta_{\mu \alpha}-\frac{q_{\mu} q_{\alpha}}{m_{l}^{2}}\right)\left(\eta_{\nu \beta}-\frac{q_{\nu} q_{\beta}}{m_{l}^{2}}\right)+\left(\eta_{\nu \alpha}-\frac{q_{\nu} q_{\alpha}}{m_{l}^{2}}\right) \\
& \times\left(\eta_{\mu \beta}-\frac{q_{\mu} q_{\beta}}{m_{l}^{2}}\right)-\frac{2}{3}\left(\eta_{\mu \nu}-\frac{q_{\mu} q_{\nu}}{m_{l}^{2}}\right)\left(\eta_{\alpha \beta}-\frac{q_{\alpha} q_{\beta}}{m_{l}^{2}}\right),
\end{aligned}
$$

where $\kappa^{2}=16 \pi G_{N}$, and $m_{l}$ is $\mathrm{KK}$ state mass. After a straightforward calculation and summing over all KK state contributions, we have

$$
\begin{aligned}
M= & -i \frac{\kappa^{2} D_{n}(s)}{4}\left[2 u \epsilon_{1} \cdot k_{1} \epsilon_{2} \cdot k_{2}+2 t \epsilon_{1} \cdot k_{2} \epsilon_{2} \cdot k_{1}+\epsilon_{1} \cdot \epsilon_{2}\right. \\
& \left.\times\left(t u-m_{h}^{4}\right)+2 m_{h}^{2}\left(\epsilon_{1} \cdot k_{1} \epsilon_{2} \cdot k_{1}+\epsilon_{1} \cdot k_{2} \epsilon_{2} \cdot k_{2}\right)\right],
\end{aligned}
$$

where $s=q^{2}, \quad t=\left(k_{1}-p_{1}\right)^{2}=\left(p_{2}-k_{2}\right)^{2}, \quad u=\left(k_{1}-p_{2}\right)^{2}$ $=\left(k_{2}-p_{1}\right)^{2}, D_{n}(s)=\Sigma_{l} i /\left(s-m_{l}^{2}\right)$. With the cutoff scale for the summation set to be the same as $M_{S}$, one has

$$
\begin{aligned}
& \kappa^{2} D_{n}(s)=\frac{16 \pi}{M_{S}^{4}}\left(\frac{s}{M_{S}^{2}}\right)^{n / 2-1}\left[\pi+i 2 I_{n}\left(M_{S} / \sqrt{s}\right)\right], \\
& I_{n}(x)= \begin{cases}-\sum_{k=1}^{n / 2-1} \frac{1}{2 k} x^{2 k}-\frac{1}{2} \ln \left(x^{2}-1\right), & \text { for } n=\text { even, } \\
-\sum_{k=1}^{(n-1) / 2} \frac{1}{2 k-1} x^{2 k-1}+\frac{1}{2} \ln \frac{x+1}{x-1}, & \text { for } n=\text { odd } .\end{cases}
\end{aligned}
$$

For $\quad M_{S}^{2} \gg s, \quad \kappa^{2} D_{n}(s)$ is approximately equal to $i\left(16 \pi / M_{S}^{4}\right) \ln \left(M_{S}^{2} / s\right)$ for $n=2$ and $i\left(16 \pi / M_{S}^{4}\right) 2 /(n-2)$ for $n>2$. There are a few different definitions of $M_{S}$ used in the literature, although in the same order of magnitude there are some numerical differences. We will use $M_{S}$ defined above for our discussions [8].

The helicity amplitudes for different photon polarizations are given by

$$
\begin{aligned}
M((1,+),(2,+)) & =M((1,-),(2,-)) \\
& =\frac{\kappa^{2} D_{n}(s)}{16} s^{2}\left(1-\frac{4 m_{h}^{2}}{s}\right) \sin ^{2} \theta,
\end{aligned}
$$

$$
M((1,+),(2,-))=M((1,-),(1,+))=0,
$$

where $\theta$ is the angle between the photon beam line and the out going Higgs boson momentum. $(1, \pm)$ and $(2, \mp)$ indicate the photon polarization vectors with $\epsilon_{1, \mu}( \pm)=\epsilon_{2, \mu}(\mp)$ $=(0, \pm 1,-i, 0) / \sqrt{2}$. The cross section for polarized photons is given by

$$
\begin{aligned}
\frac{d \sigma((1, \pm),(2, \pm))}{d \cos \theta}= & \frac{\pi}{64 M_{S}^{2}}\left(\frac{s}{M_{S}^{2}}\right)^{n+1} \\
& \times\left[\pi^{2}+4 I_{n}^{2}\left(M_{S} / \sqrt{s}\right)\right] \\
& \times \sin ^{4} \theta\left(1-\frac{4 m_{h}^{2}}{s}\right)^{5 / 2}, \\
\frac{d \sigma((1, \pm),(2, \mp))}{d \cos \theta}= & 0 .
\end{aligned}
$$

The cross section for the unpolarized photon beam, $d \sigma(s) / d \cos \theta$ is the same as $d \sigma((1, \pm),(2, \pm)) / d \cos \theta$. We will use this case for later discussions.

In $\mathrm{SM}$ the lowest order contributions to $\gamma \gamma \rightarrow H H$ occur at one-loop level. The cross section is predicted to be small. For monochromatic photon beam with $\sqrt{s}=0.5(1) \mathrm{TeV}$ and the Higgs boson mass $m_{h}$ in the range of 100-200 (100400) $\mathrm{GeV}$, the cross section $\sigma(s)$ is in the range $0.8-0.4$ $(0.6-0.5) \mathrm{fb}$ [12]. These cross sections are small and difficult to achieve experimentally although not impossible. With effects from extra dimensions the cross section can be dramatically different.

The effects of extra dimensions on the Higgs pair production at photon colliders depend on the scale $M_{S}$, the number of extra dimensions $\mathrm{n}$, the Higgs boson mass $m_{h}$, and the collider energy $\sqrt{s}$. The cross section increases rapidly with $s$ which is different than the prediction of SM where the cross section does not change dramatically with $\sqrt{s}$. The cross section decreases when $M_{S}, m_{h}$, and $n$ are increased. In our numerical analysis, we will take $M_{S}=1 \mathrm{TeV}$ and $1.5 \mathrm{TeV}$ for $\sqrt{s}=0.5 \mathrm{TeV}$, and $M_{s}=1.5 \mathrm{TeV}$ and $2 \mathrm{TeV}$ for $\sqrt{s}$ $=1 \mathrm{TeV}$ and vary $n$ up to 7 and $m_{h}$ in typical kinematic allowed ranges for illustration.

The results for $\sigma(s)$ with monochromatic photon beams are shown in Table I. From Table I, we see that for $\sqrt{s}$ $=0.5 \mathrm{TeV}$ and $m_{h}$ as high as $200 \mathrm{GeV}$, the cross sections can be as large as $274 \mathrm{fb}$ and $14 \mathrm{fb}$ for $M_{S}=1.0 \mathrm{TeV}$ and $1.5 \mathrm{TeV}$, respectively. Even with $n=7$, the cross section can still reach $11 \mathrm{fb}$ for $M_{S}=1 \mathrm{TeV}$. For $\sqrt{s}=1 \mathrm{TeV}$, the cross sections are even larger. For the worst case of $n=7$, for $m_{h}$ as large as $350 \mathrm{GeV}$ the cross section can still reach $140 \mathrm{fb}$ and $7 \mathrm{fb}$ with $M_{S}=1.5 \mathrm{TeV}$ and $2 \mathrm{TeV}$, respectively. We also analyzed the case with $\sqrt{s}=250 \mathrm{GeV}$. In this case, the cross section for $M_{S}=1.5 \mathrm{TeV}$ the cross section is small $(<0.34 \mathrm{fb})$. But the cross section can still be as large as 6.6 fb for $m_{h}=100 \mathrm{GeV}$ with $M_{S}=1 \mathrm{TeV}$. It is clear that the cross sections for Higgs pair production at photon colliders with extra dimensions can be much larger than the SM predictions. 
TABLE I. The cross section $\sigma(s)$ for $\gamma \gamma \rightarrow H H$. (a) $\sigma(s)$ (in unit fb) for $\sqrt{s}=0.5 \mathrm{TeV}$ with $M_{S}=1.0$ (1.5) TeV. (b) $\sigma(s)$ (in unit fb) for $\sqrt{s}=1.0 \mathrm{TeV}$ with $M_{S}=1.5(2.0) \mathrm{TeV}$.

\begin{tabular}{lcccccc}
\hline \hline $\begin{array}{l}m_{h}(\mathrm{GeV}) \\
(\mathrm{a})(\mathrm{fb})\end{array}$ & $n=2$ & $n=3$ & $n=4$ & $n=5$ & $n=6$ & $n=7$ \\
\hline 100 & $2280(114)$ & $941(34)$ & $461(13)$ & $249(6.09)$ & $145(3.273)$ & $91(1.98)$ \\
150 & $1155(58)$ & $477(17)$ & $233(6.66)$ & $127(3.09)$ & $74(1.66)$ & $46(1.00)$ \\
200 & $274(14)$ & $113(4.08)$ & $55(1.58)$ & $30(0.73)$ & $18(0.39)$ & $11(0.24)$ \\
$(\mathrm{b})(\mathrm{fb})$ & & & & & \\
\hline 100 & $7120(796)$ & $3760(328)$ & $2270(161)$ & $1460(87)$ & $978(51)$ & $678(32)$ \\
200 & $5100(570)$ & $2700(235)$ & $1620(115)$ & $1040(62)$ & $700(36)$ & $485(22)$ \\
350 & $1460(164)$ & $770(68)$ & $470(33)$ & $300(18)$ & $200(10)$ & $140(7)$ \\
450 & $124(14)$ & $66(6)$ & $39(3)$ & $25(1.5)$ & $17(0.9)$ & $12(0.6)$ \\
\hline \hline
\end{tabular}

High energy monochromatic photon beams may be difficult to obtain. A practical method to obtain high energy photon beams is to use a laser back-scattering technique on an electron or positron beam which produces abundant numbers of hard photons nearly along the same direction as the original electron or positron beam. The photon beam energy obtained this way is not monochromatic. The energy spectrum of the back-scattered photon is given by [14]

$$
\begin{aligned}
& F(x)=\frac{1}{D(\xi)}\left[1-x+\frac{1}{1-x}-\frac{4 x}{\xi(1-x)}+\frac{4 x^{2}}{\xi^{2}(1-x)^{2}}\right] \\
& D(\xi)=\left(1-\frac{4}{\xi}-\frac{8}{\xi^{2}}\right) \ln (1+\xi)+\frac{1}{2}+\frac{8}{\xi}-\frac{1}{2(1+\xi)^{2}},
\end{aligned}
$$

where $x$ is the fraction of the energy of the incident electron. The parameter $\xi$ is determined to be $2(1+\sqrt{2})$ by requiring that the back-scattered photon to have the largest possible energy, but does not interfere with the incident photon to create unwanted $e^{+} e^{-}$. The maximal value $x_{\max }$ can be reached by $x$ is given by $\xi /(1+\xi) \approx 0.828$.

The cross section $\sigma\left(e^{+} e^{-}\right)$, which is due to the backscattered photon beams colliding to produce Higgs pair, $e^{+} e^{-} \rightarrow \gamma \gamma \rightarrow H H$, is obtained by folding in the photon luminosities with the cross section $\sigma(s)$. We have

$$
\begin{aligned}
\sigma\left(e^{+} e^{-}\right)= & \int_{x_{1 \min }}^{x_{\max }} \int_{x_{2 \min }}^{x_{\max }} F\left(x_{1}\right) F\left(x_{2}\right) \sigma\left(x_{1} x_{2} s\right) \theta \\
& \times\left(1-\frac{4 m_{h}^{2}}{x_{1} x_{2} s}\right) d x_{1} d x_{2}
\end{aligned}
$$

with $x_{1 \text { min } 2 \text { min }}=4 m_{h}^{2} /\left(x_{\text {max }} s\right)$. Here $\sqrt{s}$ is the total energy of the $e^{ \pm}$beams.

The results for the same cases discussed for monochromatic photon beams are shown in Table II. The cross section $\sigma\left(e^{+} e^{-}\right)$is smaller than $\sigma(s)$ due to the replacement of $s$ to $x_{1} x_{2} s$. However, we still find that the cross section can be much larger than that predicted by SM. In SM for $\sqrt{s}$ $=0.5 \mathrm{TeV}$ and $1 \mathrm{TeV}$ become smaller $(<0.2 \mathrm{fb})$ compared with the case for monochromatic photon beam. With effects from extra dimensions, for $\sqrt{s}=0.5 \mathrm{TeV}$, the cross section $\sigma\left(e^{+} e^{-}\right)$can still be as large as 59 (3) fb for $m_{h}$ $=100 \mathrm{GeV}$ and $9.4(0.48)$ fb for $m_{h}=150 \mathrm{GeV}$ with $M_{S}$ $=1.0(1.5) \mathrm{TeV}$. For $\sqrt{s}=1 \mathrm{TeV}$, the cross sections are much larger. $\sigma\left(e^{+} e^{-}\right)$can be as large as 124 (14.8) fb for $m_{h}$ and as large as $200 \mathrm{GeV}$ with $M_{S}=1.5(2) \mathrm{TeV}$. For smaller $m_{h}$, the cross sections are even larger. These cross sections are much larger than SM predictions. Observations of Higgs pair events at photon colliders at a level larger than $1 \mathrm{fb}$ would be indications of effects from extra dimensions. If the effects due to extra dimensions indeed exist, production

TABLE II. The cross section $\sigma\left(e^{+} e^{-}\right)$for $e^{+} e^{-} \rightarrow \gamma \gamma \rightarrow H H$. (a) $\sigma\left(e^{+} e^{-}\right)$(in unit fb) for $\sqrt{s}$ $=0.5 \mathrm{TeV}$ with $M_{S}=1.0(1.5) \mathrm{TeV}$. (b) $\sigma\left(e^{+} e^{-}\right.$) (in unit fb) for $\sqrt{s}=1.0 \mathrm{TeV}$ with $M_{S}=1.5(2.0) \mathrm{TeV}$.

\begin{tabular}{lcccccc}
\hline \hline $\begin{array}{l}m_{h}(\mathrm{GeV}) \\
(\mathrm{a})(\mathrm{fb})\end{array}$ & $n=2$ & $n=3$ & $n=4$ & $n=5$ & $n=6$ & $n=7$ \\
\hline 100 & $59(3.03)$ & $19(0.71)$ & $7.6(0.24)$ & $3.6(0.10)$ & $1.98(0.06)$ & $1.20(0.03)$ \\
150 & $9.4(0.48)$ & $3.1(0.12)$ & $1.3(0.04)$ & $0.63(0.02)$ & $0.34(0.009)$ & $0.21(0.006)$ \\
$(\mathrm{b})(\mathrm{fb})$ & & & & & \\
\hline 100 & $271(32.5)$ & $106(10.0)$ & $50(4.00)$ & $27(1.90)$ & $15(1.03)$ & $9.5(0.62)$ \\
200 & $124(14.8)$ & $50(4.71)$ & $24(1.91)$ & $13(0.91)$ & $7.5(0.50)$ & $4.70(0.30)$ \\
350 & $2.72(0.32)$ & $1.17(0.11)$ & $0.59(0.046)$ & $0.33(0.023)$ & $0.20(0.012)$ & $0.12(0.007)$ \\
\hline \hline
\end{tabular}


of Higgs pair at photon colliders may even become practically possible. Pair production of Higgs bosons at photon colliders can provide important information about effects from extra dimensions.

We remark that the angular distribution can also provide important information about the mechanism for Higgs pair production at photon colliders. For monochromatic photon colliders the angular distribution is simply given by $d \sigma /(\sigma d \cos \theta)=15 \sin ^{4} \theta / 16$ which peaks at $\theta=\pi / 2$. For back-scattered photon beams, the angular distribution $d \sigma\left(e^{+} e^{-}\right) /\left[\sigma\left(e^{+} e^{-}\right) d \cos \theta\right]$ is more complicated. Here $\theta$ is the angle between the $e^{ \pm}$beam direction and the momentum for one of the Higgs boson in the final state. The distribution also peaks at $\theta=\pi / 2$ which is different than the effects due to SM and other new physics, such as anomalous triple Higgs boson coupling [12]. Also, the formula obtained in Eq. (5) can be used for $g g \rightarrow H H$ with a simple replace- ment of photons to gluons. Effects of extra dimensions on Higgs pair production can also be studied at hadron colliders.

To conclude, we have shown that effects due to extra dimensions can induce tree level $\gamma \gamma \rightarrow H H$ scattering. Within the allowed range for the scale $M_{S}$ the predicted cross sections can be much larger than those predicted by the standard model where $\gamma \gamma \rightarrow H H$ only occur at loop level. New physics due to extra dimensions can be tested using Higgs pair production at photon colliders. If the effects due to extra dimensions exist, production of Higgs pairs at photon colliders may even become practically possible.

Note added. After we have finished this work, we became aware of the work by Rizzo in Ref. [15] where, among other things, similar calculation was also done for $\gamma \gamma \rightarrow H H$. Our formula for this process agrees.

This work was supported in part by the National Science Council of R.O.C. under Grant No. NSC 88-2112-M-002041 .
[1] N. Arkani-Hamed, S. Dimopoulos, and G. Dvali, Phys. Lett. B 429, 263 (1998); I. Antoniadis et al., ibid. 436, 257 (1998); N. Arhani-Hamed, S. Dimopoulos, and J. March-Russell, Phys. Rev. D (to be published), hep-ph/9809124.

[2] I. Antoniadis, Phys. Lett. B 246, 377 (1990); R. Sundrum, J. High Energy Phys. 07, 001 (1999); G. Shiu and S.-H. Tye, Phys. Rev. D 58, 106007 (1998); Z. Kakushadze and S.-H. Tye, Nucl. Phys. B548, 180 (1999); I. Antoniadis, N. ArkaniHamed, S. Dimopoulos, and G. Dvali, Phys. Lett. B 436, 257 (1998).

[3] K. R. Dienes, E. Dudas, and T. Gherghetta, Phys. Lett. B 436, 55 (1998); Nucl. Phys. B537, 47 (1999); K. R. Dienes et al., ibid. B543, 387 (1999); N. Arkani-Hamed, S. Dimopoulos, and G. Dvali, Phys. Rev. D 59, 086004 (1999).

[4] G. Guidice, R. Rattazzi, and J. Wells, Nucl. Phys. B544, 3 (1999); E. Mirabelli, M. Perlestein, and M. Peskin, Phys. Rev. Lett. 82, 2236 (1999); J. Hewett, ibid. 82, 4765 (1999); P. Mathews, S. Raychaudhuri, and K. Sridhar, Phys. Lett. B 450, 343 (1999); 455, 115 (1999).

[5] T. Rizzo, Phys. Rev. D 59, 115010 (1999); hep-ph/9902273; Phys. Rev. D (to be published), hep-ph/9904380; K. Agashe and N. Deshpande, Phys. Lett. B 456, 60 (1999); K. Cheung and W.-Y. Keung, Phys. Rev. D (to be published), hep-ph/9903294; D. Atwood, S. Bar-Shalom, and A. Soni, hep-ph/9903538; C. Balazs et al., Phys. Rev. Lett. 83, 2112 (1999); A. Gupta, N. Mondal, and S. Raychaudhuri, hep-ph/9904234; H. Goldberg, hep-ph/9904318; H. Davoudasl, Phys. Rev. D 60, 084022 (1999); K.-Y. Lee et al., hep-ph/9904355; Phys. Rev. D 60, 093002 (1999).
[6] M. Graesser, hep-ph/9902310; P. Nath and M. Yamaguchi, Phys. Rev. D (to be published), hep-ph/9902323; M. Masip and A. Pomarol, ibid. 60, 096005 (1999).

[7] T. Han, J. Lykken, and R.-J. Zhang, Phys. Rev. D 59, 105006 (1999).

[8] Kingman Chang, hep-ph/9904266; Phys. Lett. B 460, 383 (1999).

[9] J. Gunion et al., Higgs Hunters' Guide (Addison-Wesley, Reading, MA, 1990); J. Gunion, A. Stange, and S. Willenbrock, in Electroweak Symmetry Breaking and New Physics at TEV, edited by T. L. Barklow, pp. 23-145, hep-ph/9602238.

[10] T. Greening, hep-ex/9903013.

[11] V. Barger, T. Han, and R. Phillips, Phys. Rev. D 38, 2766 (1988); V. Barger and T. Han, Mod. Phys. Lett. A 5, 667 (1990); K. Gaemers and F. Hoogeveen, Z. Phys. C 26, 249 (1984); W.-Y. Keung, Mod. Phys. Lett. A 2, 765 (1987); J. P. Eboli et al., Phys. Lett. B 197, 269 (1987); D. Dicus, K. Kallianpur, and S. Willenbrock, ibid. 200, 187 (1988); D. Dicus, C. Kao, and S. Willenbrock, ibid. 203, 457 (1988); A. Djouadi, J. Kalinowski, and P. Zerwas, Z. Phys. C 54, 255 (1992).

[12] G. Jikia, Nucl. Phys. B412, 57 (1994); G. Jikia and Y. Pigrogov, Phys. Lett. B 283, 135 (1992).

[13] L.-Z. Sun and Y.-Y. Liu, Phys. Rev. D 54, 3563 (1996).

[14] I. Ginzburg et al., Nucl. Instrum. Methods Phys. Res. 205, 57 (1983); 219, 5 (1984); Pis'ma Zh. Éksp. Teor. Fiz. 34, 514 (1981) [ JETP Lett. 34, 491 (1981)]; V. Telnov, Nucl. Instrum. Methods Phys. Res. A 294, 72 (1990).

[15] T. Rizzo, Phys. Rev. D 60, 075001 (1999). 